Artículo

\title{
Comportamiento de líneas élite de cártamo de alta productividad y calidad de aceite en el Valle del Yaqui, Sonora
}

\author{
Néstor Alberto Aguilera-Molina ${ }^{1}$ \\ Agustín Hernández-Juárez ${ }^{1 \S}$ \\ Lope Montoya-Coronado ${ }^{2}$ \\ Luis Alberto Aguirre-Uribe ${ }^{1}$ \\ Ernesto Cerna-Chávez ${ }^{1}$ \\ Jerónimo Landeros-Flores ${ }^{1}$ \\ ${ }^{1}$ Universidad Autónoma Agraria Antonio Narro-Departamento de Parasitología. Calzada Antonio Narro \\ núm. 1923, Buenavista, Saltillo, Coahuila, México. (aguilera.nestor@inifap.gob.mx; \\ luisaguirreu@yahoo.com.mx; jabaly1@yahoo.com). CP. 25315. ${ }^{2}$ Campo Experimental Norman E. \\ Borlaug-INIFAP. Calle Dr. Norman E. Borlaug km 12, Valle del Yaqui, Cajeme, Ciudad Obregón, Sonora, \\ México. CP. 8500. (montoya.lope@inifap.gob.mx). \\ ${ }^{\S}$ Autor para correspondencia: chinoahj14@ hotmail.com.
}

\section{Resumen}

El cártamo Carthamus tinctorius L. es un cultivo que ha sido adoptado por un gran número de productores, particularmente en el Noroeste del país, debido a su gran adaptabilidad y a su poco requerimiento hídrico, a causa de las grandes demandas que presenta la industria de oleaginosas en el país. Desde la década de los 70's se ha trabajado para generar materiales que sean altamente productivos, con buen porcentaje y calidad de aceite y con características de resistencia a las principales enfermedades presentes en la región (falsa cenicilla y roya de la hoja). Por esto el objetivo de este trabajo fue evaluar el comportamiento de las líneas elite de cártamo generadas por el programa de mejoramiento genético en la región (23 oleicas y 1 linoleica). Dichas líneas se establecieron durante los ciclos 2017-2018 y 2018-2019. Se evaluó su comportamiento agronómico bajo las condiciones presentes del Valle del Yaqui. Se encontraron líneas del tipo oleico que superaron a los testigos Ciano ol y Chey ol, variedades que se siembran ampliamente en la región. Además, se encontró que la línea del tipo linoleico presentó mejores características agronómicas, producción y contenido de aceite que el testigo RC-1002 lo cual lleva a concluir que dentro de las líneas evaluadas hay materiales potenciales para ser liberados como variedad.

Palabras clave: fitomejoramiento, oleaginosas, producción.

Recibido: enero de 2021

Aceptado: marzo de 2021 


\section{Introducción}

El cártamo Carthamus tinctorius L. (Asteraceae) es una especie que se cultiva a nivel mundial, es originaria de medio oriente y tradicionalmente fue utilizada para producir tintes a partir de sus pétalos. En la actualidad se utiliza tanto para fines medicinales como alimenticios (Machewad et al., 2012; Asgarpanah y Kasemivash, 2013). La mayor parte de la producción de grano se utiliza para la extracción de aceite, el cual es de muy buena calidad y hay de dos tipos los que contienen una alta concentración de ácido linoleico y los que contienen ácido oleico, los cuales traen beneficios para la alimentación de la población en general (Borbón et al., 2019).

Los principales países productores de cártamo a nivel mundial son: Kazajistán, Rusia, México y Estados Unidos, con una producción de 196 000, 193 000, 96000 y 90000 ton respectivamente (FAOSTAT, 2019). En el mundo generalmente se cultiva en terrenos escasamente fértiles o de poco rendimiento debido a las condiciones 'rústicas' que presenta esta especie. Sin embargo, en el sur de Sonora debido a las condiciones de déficit hídrico, el cártamo ha sido adoptado por una gran numero de agricultores, los cuales en su mayoría lo cultivan en terrenos de buena calidad (Montoya, 2010). Y aunque México es uno de los principales productores mundiales del cultivo, durante 2016 solo se logró cubrir $50.5 \%$ de semilla de cártamo requerido por la industria nacional (Ramos et al., 2019). Situación que es constante desde la década de los 90’s (Chanda et al., 1990).

En México el cártamo es cultivado principalmente para la producción de aceite comestible y en los últimos años se han llevado a cabo experimentos para evaluar su uso como forraje ya sea ensilado o en fresco para ganado bovino y caprino (Landau et al., 2004; Reta et al., 2017). La producción de esta especie se concentra en el norte del país dentro de los estados de: Sonora, Baja California y Tamaulipas, con una superficie de 15 000, 2600 y 1910 ha sembradas.

El cultivo de cártamo genera $0.11 \%$ del PIB agrícola nacional y cubre $15.31 \%$ de la producción de oleaginosas nacional (SAGARPA, 2017; SIAP, 2021), su cosecha está completamente mecanizada y los lugares donde se presenta la mayor productividad es donde se irriga, como es el caso de Sonora, donde la mayor parte de esta área está concentrada dentro de los Valles del Yaqui y Mayo (Silveira et al., 2009; Ramírez et al., 2017). Siendo estas las regiones más importantes del estado, en el Yaqui se establecen dos ciclos en el año, donde en el otoñoinvierno los cultivos más importantes son el trigo y el maíz y durante el verano se establece un área importante de soya y maíz dependiendo de la disponibilidad de por parte del sistema de presas del Rio Yaqui (Cerutti, 2019).

El cártamo es un cultivo de gran importancia en el noroeste de México, gracias a su capacidad de producción con bajos niveles de agua, en comparación con el cultivo de trigo, el cual predomina en la región; sin embargo, debido a los efectos del cambio climático cada vez se registran menos captaciones de agua en el sistema de presas del Rio Yaqui (Minjares et al., 2010), es importante tomar en cuenta que una parte del agua contenida en estos reservorios es utilizada para el consumo humano, por las localidades de la cuenca del Rio Yaqui, además la insistencia por los productores de sembrar trigo ha reducido cada vez el área de siembra de cultivos de verano como: soya, sorgo, entre otros. Lo cual ha ocasionado déficit hídrico para el cultivo de trigo (Paquini et al., 2016). 
En el Valle del Mayo esta problemática es más evidente debido que esta región no se irriga con el sistema de presas, sino con la presa del Mocúzari la cuales es abastecida con el caudal proveniente del Río Mayo, que no da abasto para mantener el área agrícola, por lo cual es común que se registren programas de gobierno que impulsen la siembra del cultivo de cártamo el cual es óptimo debido a su bajo requerimiento de agua (Khalili et al., 2014; Martínez et al., 2016; Singh et al., 2016).

El programa de mejoramiento genético cártamo inicio desde la década de los 70's con el objetivo de identificar materiales que se adapten a las condiciones de la región y que presenten calidad y productividad (Quilantán, 1978). A lo largo del tiempo se ha trabajado para generar materiales que cubran las necesidades de los productores y gracias a esta actividad se han logrado liberar variedades con resistencia a distintas enfermedades de importancia mundial como la roya del cártamo (Puccinia carthami) y alternaria (Alternaria carthami) (Montoya y Ochoa, 2006).

Durante el ciclo agrícola 2000-2001 en los Valles del Yaqui y Mayo se presentó la enfermedad conocida como falsa cenicilla ocasionada por el hongo $R$. carthami (Hostert et al., 2006) la cual durante el ciclo 2003-2004 presentó el mayor daño registrado, durante este ciclo se habían establecido 107833 ha en el Valle el área más grande en los últimos 15 años, de las cuales se reportaron siniestradas 2410 a causa de la enfermedad (SIAP 2021), se estimó que en esta zona la falsa cenicilla ocasionó una pérdida por alrededor de 133000 t equivalentes a 440 millones de pesos, como resultado de esto se inició la generación de variedades que presentaran características de resistencia y los primeros resultados de este trabajo se vieron reflejados en 2008 cuando se liberaron las primeras variedades tolerantes a esta enfermedad (Montoya et al., 2008; Montoya, 2010; Borbón et al., 2011; JLSVVY, 2019).

Con el paso del tiempo el área de siembra del cultivo ha variado en el estado de Sonora, a partir de 2010 el año con la mayor superficie fue 2012 con 56452 ha, seguido de 2011 con 45202 ha, la constante presión de la enfermedad, así como la disponibilidad de agua y el precio del cultivo han propiciado que durante 2019 se presentara la superficie más baja con solo 13397 ha sembradas en el estado (Montoya et al., 2008; Ávila et al., 2014; SIAP 2021). Además, las condiciones climáticas han estado cambiando y es necesario continuar generando materiales que se adapten a este escenario (Padmavathi y Virmani, 2013).

Dentro del cultivo de cártamo existen dos tipos: los que producen un aceite de alto porcentaje de monoinsaturados y ácido oleico, y aquellas de con alta concentración de ácidos poliinsaturados y acido linoleico (Zapata, 2010; SAGARPA, 2017). Históricamente dentro del programa de mejoramiento genético se generaban variedades que contenían aceite del tipo linoleico, debido a que era lo requerido por la industria (Montoya et al., 2008; Borbón et al., 2011); sin embargo, en los últimos años atendiendo las necesidades de la industria de los aceites comestibles se hizo énfasis en la generación de materiales de cártamo con alto contenido de ácido oleico (Montoya et al., 2008) debido a las propiedades benignas que tiene sobre la alimentación humana (Montes et al., 2016).

Pero aún se mantiene la investigación de materiales del tipo linoleico, con el propósito de prevenir posibles necesidades de estos materiales en el futuro próximo, por parte de los agricultores y la industria (Montoya et al., 2008). Por tanto, el objetivo de la presente investigación fue determinar el comportamiento agronómico de las líneas elite del tipo oleico y linoleico generadas por el programa de mejoramiento genético y además identificar posibles materiales candidatos a ser liberados como nuevas variedades. con base a su potencial productivo, calidad y tolerancia a las enfermedades presentes en la región. 


\section{Materiales y métodos}

El presente trabajo se llevó a cabo dentro de las instalaciones del Campo Experimental Norman E. Borlaug ubicado en el block 910 del Valle del Yaqui, durante los ciclos otoño-invierno 2016-17 y otoño-invierno 2017-2018 donde se aplicó un riego previo para poder sembrarlas a tierra venida, con fechas de siembra de: 20 de diciembre de 2017 y el 24 de enero de 2019 respectivamente. Se establecieron 24 líneas elite (Cuadro 1), provenientes del programa de mejoramiento de cártamo (23 oleicas, 1 linoleica), seleccionadas por sus buenas características agronómicas, así como su buena producción y calidad de aceite, también por presentar tolerancia a la falsa cenicilla y 3 testigos RC-1002 (linoleico), Chey ol y Ciano ol (Oleico), variedades que se siembran ampliamente en la región. Todos los materiales fueron generados por el programa mediante métodos tradicionales de mejoramiento (Pedigree) y selección. A lo largo de su desarrollo fueron sometidas a diferentes tipos de estrés y se realizaron selecciones recurrentes para poder llegar a obtener dichos materiales.

\section{Cuadro 1. Líneas y variedades utilizados para el desarrollo del presente estudio.}

\begin{tabular}{cccccc}
\hline Material & Origen & Tipo & Material & Origen & Tipo \\
\hline 0272-12-3-5-OY & Línea élite & Oleico & CC1644-1-2-0Y & Línea élite & Oleico \\
0272-3-2 & Línea élite & Oleico & CCC-1561 & Línea élite & Oleico \\
C-27-1Y-2Y(A/N) & Línea élite & Oleico & CCC-1564-1-1-1-1-0Y & Línea élite & Oleico \\
C-32-0Y & Línea élite & Oleico & CCC-1633-1-1-1-0Y & Línea élite & Oleico \\
C-54-1-OY & Línea élite & Oleico & CCC-1651-1-1-1-2-0Y & Línea élite & Oleico \\
C-56-1Y-1Y & Línea élite & Oleico & CCC-1651-1-1-1-2Y-1Y & Línea élite Oleico \\
C-61-1Y-1Y & Línea élite & Oleico & CCC-1672-1-1-1-1 & Línea élite Oleico \\
C-63-0Y & Línea élite & Oleico & S-518-SEL-PPR'SS10 (A/N) & Línea élite Oleico \\
C-65-0Y & Línea élite & Oleico & CIANO-OL/CC1658-1-0Y & Línea élite Oleico \\
C-72-0Y & Línea élite & Oleico & S-518-SEL-PPR'SS10 (A/N) & Línea élite Oleico & Ole \\
CC-14-31-5-1-OY. 1-2-0Y Línea élite & Oleico & CHEY-OL & Variedad Oleico \\
CC1567-1-1-0Y-1C & Línea élite & Oleico & CIANO-OL & Variedad Oleico \\
CC-15-76-1-1-0Y-1C & Línea élite & Oleico & RC-1002 & Variedad Linoleico \\
CC1631-1-1-1-1 & Línea élite Oleico & & & \\
CC1635-1-1-1-1Y & Línea élite Linoleico & & & \\
\hline
\end{tabular}

Se estableció un diseño experimental totalmente al azar con cuatro repeticiones, en una parcela de cuatro surcos a $80 \mathrm{~cm}$ de separación y una longitud de $6 \mathrm{~m}\left(19.2 \mathrm{~m}^{2}\right)$. A estos materiales se les dio el manejo agronómico recomendado por el INIFAP en la región, el cual consistió en una fertilización de 150-52-00 (N-P-K), además se realizaron dos riegos de auxilio durante la etapa de elongación de tallo y la de formación de botones florales, se aplicaron insecticidas para el control de insectos y no se llevó a cabo ninguna aplicación para el control de enfermedades. 
Se llevaron a cabo muestreos semanales cuando la planta pasó la etapa de roseta y hasta el inicio de floración, en busca de las lesiones características causadas por la falsa cenicilla $R$. carthami. Y la roya de la hoja causada por Puccinia carthami. De igual forma, se tomaron datos climáticos de la estación automatizada ubicada dentro del block 910, registrando las variables de: temperatura promedio, máxima y mínima, humedad relativa y precipitación (REMAS, 2019).

En cuanto a variables agronómicas se registraron las variables de: altura de planta, donde se tomó la medida de tres plantas por repetición con ayuda de un estadal y se promediaron los valores. Para determinar la madurez fisiológica se contabilizaron los días transcurridos desde la siembra hasta que la planta presentara síntomas de senescencia. La incidencia y severidad de la falsa cenicilla fue determinada utilizando la escala propuesta por Ramírez et al. (2011). La cual consistió en dividir visualmente en tercios inferior, medio y superior. Para evaluar la severidad se utilizó una escala arbitraria y continua de 1 a 100\%, de acuerdo con la estimación visual de área foliar afectada por los síntomas en cada hoja muestreada. Se tomaron datos de una hoja por estrato por planta.

Para la variable rendimiento de cosecharon $4 \mathrm{~m}$ de la parcela dejando $1 \mathrm{~m}$ de cabecera en los bordes, para después ser procesados con una trilladora de tipo 'Pullman', pesar el grano obtenido y convertirlo a $\mathrm{kg} \mathrm{ha}^{-1}$. A los valores obtenidos de esta variable se les realizo un análisis de varianza y una prueba de medias con diferencia mínima significativa (DMS) para determinar significancias estadísticas. Del producto cosechado se tomó una muestra de $100 \mathrm{~g}$ de grano y fue procesada con un analizador de aceite de la FOSS para determinar el contenido de aceite.

\section{Resultados y discusión}

Durante los dos ciclos de evaluación las condiciones climáticas no fueron favorables para el desarrollo de la falsa cenicilla; sin embargo; durante el primer ciclo de evaluación 2017-2018 se presentaron síntomas leves de la enfermedad en las líneas, C-54-1-OY, C-27-1Y-2Y(A/N), S-518SEL-PPR'SS10 (A/N) los cuales no llegaron ni al 5\% del área foliar afectada según la escala propuesta por Ramírez et al., 2011 y durante el segundo ciclo de evaluación no se presentaron síntomas de la enfermedad en ninguna de las líneas, ni en los testigos regionales

Al respecto Ramírez et al. (2011); Singh et al. (2019) mencionan que $R$. carthami es una enfermedad que requiere condiciones específicas de temperatura y humedad, además de que los materiales evaluados en el estudio fueron seleccionados aparte de sus características agronómicas y de calidad de aceite, por presentar resistencia a dicha enfermedad. Solo se llevó a cabo una aplicación para el control del complejo de insectos chupadores (Lygus lineolaris y Nezara viridula) presentes durante el periodo de formación de botones y floración. Dicha aplicación se realizó con un avión, debido a la imposibilidad de hacerla terrestre a causa de la altura del cultivo. En el Cuadro 2 se observó la variabilidad en cuanto a las características agronómicas de los materiales evaluados.

Las diferencias entre las variables de días a madurez y altura, durante los dos ciclos evaluados se debe a la distinta fecha de siembra, la primera del 20 de diciembre de 2019 y la otra del 24 de enero de 2019. Durante ambos periodos de siembra todos los materiales cumplieron su ciclo vegetativo antes del periodo de tiempo de 140 a 150 días (Navejas et al., 2008; Nikabadi et al., 2008); Padmavathi y Virmani, 2013; Ávila et al., 2014), para el Noroeste. Incluso durante el segundo año de evaluación algunas líneas como: C-56-1Y-1Y, CCC-1561, CCC-1672-1-1-1-1. 
Cuadro 2. Datos agronómicos de las líneas elite de cártamo ciclos 2017-2018, 2018-2019. Ciudad Obregón Sonora.

\begin{tabular}{|c|c|c|c|c|}
\hline \multirow{2}{*}{ Genotipo } & \multicolumn{2}{|c|}{ Días a madurez } & \multicolumn{2}{|c|}{ Altura $(\mathrm{cm})$} \\
\hline & $2017-2018$ & 2018-2019 & $2017-2018$ & 2018-2019 \\
\hline $0272-12-3-5-\mathrm{OY}$ & 133 & 105 & 137 & 101 \\
\hline $0272-3-2$ & 135 & 104 & 142 & 99 \\
\hline $\mathrm{C}-27-1 \mathrm{Y}-2 \mathrm{Y}(\mathrm{A} / \mathrm{N})$ & 137 & 102 & 137 & 98 \\
\hline $\mathrm{C}-32-0 \mathrm{Y}$ & 138 & 109 & 146 & 97 \\
\hline C-54-1-OY & 135 & 106 & 139 & 96 \\
\hline C-56-1Y-1Y & 138 & 96 & 138 & 95 \\
\hline C-61-1Y-1Y & 138 & 100 & 134 & 100 \\
\hline C-63-0Y & 138 & 110 & 146 & 101 \\
\hline C-65-0Y & 138 & 105 & 149 & 95 \\
\hline C-72-0Y & 138 & 107 & 143 & 99 \\
\hline CC-14-31-5-1-OY. 1-2-0Y & 135 & 137 & 165 & 102 \\
\hline CC1567-1-1-0Y-1C & 133 & 105 & 142 & 99 \\
\hline CC-15-76-1-1-0Y-1C & 133 & 103 & 144 & 95 \\
\hline CC1631-1-1-1-1 & 138 & 101 & 134 & 102 \\
\hline CC1635-1-1-1-1Y & 138 & 106 & 151 & 93 \\
\hline CC1644-1-2-0Y & 137 & 113 & 146 & 107 \\
\hline CCC-1561 & 137 & 93 & 146 & 91 \\
\hline CCC-1564-1-1-1-1-0Y & 137 & 112 & 141 & 96 \\
\hline CCC-1633-1-1-1-0Y & 135 & 111 & 146 & 97 \\
\hline CCC-1651-1-1-1-2-0Y & 135 & 116 & 145 & 110 \\
\hline CCC-1651-1-1-1-2Y-1Y & 138 & 113 & 153 & 105 \\
\hline CCC-1672-1-1-1-1 & 138 & 97 & 141 & 92 \\
\hline S-518-SEL-PPR'SS10 (A/N) & 137 & 101 & 143 & 98 \\
\hline CIANO-OL/CC1658-1-0Y & 138 & 103 & 165 & 106 \\
\hline S-518-SEL-PPR'SS10 (A/N) & 137 & 101 & 143 & 98 \\
\hline CHEY-OL & 138 & 137 & 127 & 94 \\
\hline CIANO-OL & 135 & 137 & 163 & 102 \\
\hline RC-1002 & 135 & 137 & 131 & 96 \\
\hline
\end{tabular}

Lograron completar el ciclo antes de los 100 días superando por mucho lo reportado anteriormente coincidiendo a lo reportado por Bellé et al. (2012); Navejas et al. (2014); Ramonda (2019) los cuales mencionan que la fecha de siembra es un factor muy importante para el desarrollo del cultivo, ya que plantas establecidas en fechas tempranas tienden a alargar su ciclo y producir portes exuberantes en comparación con plantas de fechas tardías e intermedias que acortan su ciclo vegetativo y su altura. De acuerdo con Montoya (2010); Golkar et al. (2012) materiales con características de precocidad, de un porte compacto, contenido de aceite por encima de $38 \%$ y una buena producción, son favorables para ser liberadas como variedades comerciales. 
La producción potencial de aceite en el cultivo de cártamo es una característica extremadamente compleja y es el resultado de múltiples funciones del crecimiento y desarrollo, las cuales están controladas por la interacción del genotipo y el ambiente (Kuruvadi et al., 1993; Ashrafi y Razmjoo, 2010). A lo largo del tiempo siempre ha sido un objetivo primordial de los fitomejoradores de cártamo el introducir esta característica a todos los materiales que se generan ya que, aunque se le pueden dar distintos usos al cultivo, su vocación natural es la producción de aceite para consumo humano y ocasionado por la búsqueda de nuevas fuentes de energía se ha investigado su potencial para producir biocombustible (Ullah y Bano, 2011; Vosoughkia et al., 2012; Eryilmaz y Yesilyurt, 2016).

A lo largo del tiempo el programa de mejoramiento genético se había dedicado a generar materiales del tipo linoleico, los cuales se han cultivado en diferentes países de medio oriente como Irán e India (Pourad, 2008; Mahboobeh et al., 2011), en los últimos años la investigación ha disminuido en este apartado. Dentro de los materiales evaluados de este tipo se encuentra la línea: CC1635-11-1-1Y la cual ha presentado valores de producción y porcentaje de aceite superior al testigo del tipo Linoleico RC-1002 (Cuadro 3) (Montoya 2008). Y a otras variedades comerciales del mismo tipo como son la variedad 'Promesa y Guayalejo' de reciente liberación (Valadez y Cervantes, 2013; Valadez y Gutiérrez, 2017).

Cuadro 3. Producción y calidad de líneas elite de cártamo ciclos 2017-2018, 2018-2019. Ciudad Obregón Sonora.

\begin{tabular}{|c|c|c|c|c|c|}
\hline \multirow{2}{*}{ Genotipo } & \multicolumn{2}{|c|}{$\%$ de aceite } & \multicolumn{3}{|c|}{ Rendimiento $\left(\mathrm{kg} \mathrm{ha}^{-1}\right)$} \\
\hline & 2017-2018 & 2018-2019 & $2017-2018$ & $2018-2$ & 2019 \\
\hline 0272-12-3-5-OY & 34.82 & 36.43 & 1575 abcde & 1764 & $a b c$ \\
\hline $0272-3-2$ & 38.01 & 37.66 & $1968 \mathrm{ab}$ & 1216 & $\mathrm{c}$ \\
\hline $\mathrm{C}-27-1 \mathrm{Y}-2 \mathrm{Y}(\mathrm{A} / \mathrm{N})$ & 37.71 & 37.44 & 1548 abcde & 1849 & $a b$ \\
\hline C-32-0Y & 37.44 & 37.1 & 1473 bcde & 1329 & $\mathrm{bc}$ \\
\hline C-54-1-OY & 37.28 & 37.62 & 1697 abcde & 1367 & $\mathrm{bc}$ \\
\hline C-56-1Y-1Y & 37.8 & 37.65 & 1629 abcde & 1271 & $\mathrm{bc}$ \\
\hline C-61-1Y-1Y & 37.21 & 38.72 & $1314 \mathrm{de}$ & 1193 & $\mathrm{c}$ \\
\hline C-63-0Y & 39.44 & 38.1 & 1665 abcde & 1994 & $\mathrm{a}$ \\
\hline C-65-0Y & 38.97 & 38.27 & 1670 abcde & 1292 & $a b c$ \\
\hline C-72-0Y & 36.49 & 36.13 & 2028 a & 1277 & $\mathrm{bc}$ \\
\hline CC-14-31-5-1-OY .... 1-2-0Y & 39.76 & 43.32 & 1717 abcde & 1703 & $a b c$ \\
\hline CC1567-1-1-0Y-1C & 38.22 & 37.85 & 1479 abcde & 1398 & $a b c$ \\
\hline CC-15-76-1-1-0Y-1C & 36.77 & 37.66 & $1200 \mathrm{de}$ & 1174 & $\mathrm{c}$ \\
\hline CC1631-1-1-1-1 & 38.42 & 36.78 & $1214 \mathrm{de}$ & 1246 & $\mathrm{bc}$ \\
\hline CC1635-1-1-1-1Y & 40.45 & 39.17 & 1436 bcde & 1215 & $\mathrm{c}$ \\
\hline CC1644-1-2-0Y & 36.76 & 36.68 & 1362 cde & 1213 & $\mathrm{c}$ \\
\hline CCC-1561 & 38.16 & 36.93 & $1704 \mathrm{abcd}$ & 1432 & $a b c$ \\
\hline CCC-1564-1-1-1-1-0Y & 37.13 & 37.68 & $1139 \mathrm{e}$ & 1554 & $a b c$ \\
\hline CCC-1633-1-1-1-0Y & 37.25 & 37.69 & 1488 abcde & 1328 & $\mathrm{bc}$ \\
\hline CCC-1651-1-1-1-2-0Y & 37.96 & 37.95 & $1234 \mathrm{de}$ & 1323 & $\mathrm{bc}$ \\
\hline
\end{tabular}




\begin{tabular}{|c|c|c|c|c|c|}
\hline \multirow{2}{*}{ Genotipo } & \multicolumn{2}{|c|}{$\%$ de aceite } & \multicolumn{3}{|c|}{ Rendimiento $\left(\mathrm{kg} \mathrm{ha}^{-1}\right)$} \\
\hline & $2017-2018$ & 2018-2019 & $2017-2018$ & $2018-$ & 2019 \\
\hline CCC-1651-1-1-1-2Y-1Y & 37.95 & 36.65 & 1647 abcde & 1471 & abc \\
\hline CCC-1672-1-1-1-1 & 42.16 & 37.95 & 1672 abcde & 1217 & $\mathrm{c}$ \\
\hline S-518-SEL-PPR'SS10 (A/N) & 38.22 & 36.43 & 1634 abcde & 1183 & $\mathrm{c}$ \\
\hline CIANO-OL/CC1658-1-0Y & 37.02 & 36.88 & $1276 \mathrm{ded}$ & 1288 & $\mathrm{bc}$ \\
\hline S-518-SEL-PPR'SS10 (A/N) & 38.22 & 36.43 & 1634 abcde & 1183 & $\mathrm{c}$ \\
\hline CHEY-OL & 36.4 & 36.24 & 1614 abcde & 1193 & $\mathrm{c}$ \\
\hline CIANO-OL & 37.76 & 37.24 & 1676 abcde & 1500 & abc \\
\hline RC-1002 & 40.77 & 37.37 & $1892 \mathrm{abc}$ & 1319 & bc \\
\hline
\end{tabular}

$\alpha=95 \%$; DMS 2017-2018= 571.244; DMS 2018-2019=624.522.

En los últimos años la investigación se ha orientado a generar materiales con alto contenido de ácido oleico. Dentro de las líneas evaluadas se pueden encontrar algunas que presentan valores de producción y contenido de aceite superior al de los testigos Ciano Ol y Chey Ol. de entre estas se pueden encontrar: CC-14-31-5-1-OY 1-2-0Y, y CCC-1672-1-1-1-1 con un porcentaje de aceite de $43 \%$ y de $42 \%$ respectivamente. Superando a ampliamente a los testigos anteriormente mencionados lo cuales presentan solo 37 y 36\% de aceite (Montoya et al., 2008; Ávila et al., 2017).

\section{Conclusiones}

Dentro de los materiales evaluados hay líneas del tipo oleico como: CC-14-31-5-1-OY. 1-2-0Y y CCC-1672-1-1-1-1. que presentan características agronómicas que las hacen posibles candidatas a ser liberadas como variedades; sin embargo, es necesario hacer evaluaciones en las distintas regiones productoras del noroeste de México para conocer su comportamiento bajo las distintas características climáticas presentes en cada zona.

Aunque la investigación con respecto a materiales linoleicos ha disminuido, aún se siguen generando materiales con el objetivo de satisfacer posibles necesidades del productor y de la industria. Como es el caso de la línea CC1635-1-1-1-1Y la cual ha logrado superar al testigo linoleico RC-1002. Y lo cual la coloca como candidata a ser liberada como variedad en caso de que los materiales del tipo linoleico sean requeridos.

Los resultados de este trabajo demuestran que dentro del programa de mejoramiento genético del cultivo de cártamo se han obtenido buenos resultados y si bien, la generación de materiales que presenten resistencia a enfermedades y alta productividad y contenido de aceite es un incentivo para que los productores se decidan a cultivar cártamo, la verdad es que el factor determinante para que la superficie de cártamo se incremente es el precio.

\section{Agradecimientos}

Los autores(as) agradecen al Consejo Nacional de Ciencia y Tecnología (CONACYT) por el apoyo brindado al desarrollo de este trabajo y al Programa de Mejoramiento Genético del cultivo de cártamo del Instituto de Investigaciones Forestales Agrícolas y Pecuarias (INIFAP) por las facilidades otorgadas en la elaboración de este estudio. 


\section{Literatura citada}

Asgarpanah, J. and Kasemivash, N. 2013. Phytochemistry, pharmacology and medicinal properties of Carthamus tinctorius L. Chinese J. Integrative Medicine. 19(2):153-159. https://doi.org/10.1007/s11655-013-1354-5.

Ashrafi, E. and Razmjoo, K. 2010. Effect of irrigation regimes on oil content and composition of safflower (Carthamus tinctorius L.) cultivars. J. American Oil Chemists Society. 87(5):499-506. https://doi.org/10.1007/s11746-009-1527-8.

Ávila, C. E. J. I.; Alvarado, P. M.; Camarillo, P. X. M.; Ochoa, E. y Montoya, L. C. 2014. Descripción de variedades de cártamo para el Valle de Mexicali. B. C. INIFAP-CIRNOCEMEXI. Mexicali, Baja California. Folleto para productores núm. 62. 12 p.

Ávila, C. E. X. M.; Ochoa, E. L.; Montoya, C. N. A.; Aguilera, M. A.; Borbón, G. y Alvarado, J. I. P. 2017. Chey-ol: nueva variedad de cártamo oleica para el noroeste de México. Rev. Mex. Cienc. Agríc. 8(5):1209-1212.

Bellé, A. R. E.; Kersting-Da, R. F. A. A.; Londero, B. M.; Neuhaus, M. and Teixeira, N. S. 2012. Safflower grown in diferents sowing dates and plant densities. Ciencia Rural. 42(12):2145-2152. https://doi.org/10.1590/S0103-84782012005000106.

Borbón, G. A. L.; Montoya, C. X. M.; Ochoa, E. N. A.; Aguilera, M. E.; Ávila, C. y Cota, C. I. B. 2019. SEMAY OL, Nueva variedad de cártamo oleica. Rev. Fitotec. Mex. 42(1):83-85.

Borbón, G. A. X. M.; Ochoa, E. L.; Montoya, C. J.; Pérez, M. y García, M. G. C. 2011. CIANOLIN: nueva variedad de cártamo linoleica. Rev. Mex. Cienc. Agríc. 2(5):791-794.

Cerutti, M. 2019. Trigo y revolución verde en el noroeste de México mundo agrario. 20(43):1930-1970. Doi: https://doi.org/10.24215/15155994e103.

Chanda, M. G. S.; Muñoz, V. R. D. y García, P. 1990. San José 89: nueva variedad de cártamo. INIFAP-CIFAES-CEVY. Cd. Obregón Sonora. Folleto para técnico núm. 16. 16 p.

Eryilmaz, T. and Yesilyurt, M. K. 2016. Influence of blending ratio on the physicochemical properties of safflower oil methyl ester-safflower oil, safflower oil methyl ester-diesel and $\begin{array}{llll}\text { safflower } & \text { oil-diesel. } & \text { Renewable } & \text { Energy. }\end{array}$ https://doi.org/10.1016/j.renene.2016.04.009.

FAOSTAT. 2019. Food and Agriculture Organization of the United Nations Crops. Food and Agriculture Organization of the United Nations. Rome, Italy. http://www.fao.org/faostat/en/\#data/QC/visualize.

Golkar, P. A.; Arzani. and Rezael, A. 2012. Genetic analysis of agronomic traits in safflower (Carthamus tinctorius L.). Notulae Botanicae Horti Agrobotanici Cluj-Napoca. 40(1):276-281. Doi: https://doi.org/10.15835/nbha4017209.

Hostert, N. D. C. L.; Blomquist, S. L.; Thomas, D. G.; Fogle, D. G. and Davis, R. M. 2006. First report of Ramularia carthami, causal agent of Ramularia leaf spot of safflower, in California. Plant Disease. 90(9):1260-1261. https://doi.org/10.1094/PD-90-1260C.

JLSVVY. 2019. Junta Local de Sanidad Vegetal del Valle del Yaqui. Falsa cenicilla del cártamo. http://jlsvyaqui.org.mx/?page_id=122.

Khalili, M. A.; Pour-Aboughadareh, M. R.; Naghavi, M. R. and Mohammad-amini, E. 2014. Evaluation of drought tolerance in safflower genotypes based on drought tolerance indices. Notulae Botanicae Horti Agrobotanici Cluj-Napoca. 42(1):214-218. Doi: 10.15835/nbha4219331. 
Kuruvadi, S. R. Aguilera, R. y López, A. B. 1993. Coeficiente de sendero para aceite y sus componentes asociados en cártamo bajo ambientes de riego y temporal. Agraria, Revista Científica UAAAN. 9(2):116-125.

Landau, S. S.; Friedman, S.; Brenner, I.; Bruckental, Z. G.; Weinberg, G.; Ashbell, Y.; Hen, L.; Dvash, Y. and Leshem, Y. 2004. The value of safflower (Carthamus tinctorius) hay and silage grown under Mediterranean conditions as forage for dairy cattle. Livestock Production Science. 88(3):263-271. https://doi.org/10.1016/j.livprodsci.2003.11.011.

Machewad, G. M. P.; Ghatge, V.; Chappalwar, B.; Jadhav, B. and Chappalwar, A. 2012. Studies on extraction of safflower pigments and its utilization in ice cream. J. Food Proc. Technol. 3(6):3-8. Doi: 10.4172/2157-7110.1000172.

Mahboobeh, V. M.; Ghavamib, M.; Gharachorloo, M.; Sharrifmoghaddasi, M. and Omidi A. A. 2011. Lipid composition and oxidative stability of oils in safflower (Carthamus tinctorius L.) seed varieties grow in Iran. Adv. Envirom. Biol. 5(5):897-902.

Martínez, C. D. A. J.; Chávez, M. A.; Bustamante, G. O. L.; Palacios, V. M. L.; De-Isla, B. y Tijerina, L. C. 2016. Variación espacial de la calidad del agua para uso agrícola del acuífero costero del Valle del Mayo, Sonora, México. Hidrobiológica. 26(1):109-119.

Minjarez, L. J. L. B.; Valdés, J. R. F.; Salmon, C. and Oroz, L. A R. 2010. Sustainable planning, management, and evaluation of water resources in irrigation District No. 041, Río Yaqui, Mexico. Water technology and sciences, formerly hydraulic engineering in Mexico. 1(1):137-151.

Montes, O. N. I.; Millar, M. R.; Provoste, L. N.; Martínez, M. D.; Fernández, Z. G.; Morales, I. y Valenzuela, R. B. 2016. Absorción de aceite en alimentos fritos. Revista Chilena de Nutrición. 43(1):87-91. http://dx.doi.org/10.4067/S0717-75182016000100013.

Montoya, C. L. C. M.; Armenta, C. J. A.; Ramírez, A. R.; Valenzuela, B. I.; Armenta, C. F.; Cabrera, C. M J.; Beltrán, F. R. A.; Lagarda, G. A.; Borbón, G. y Ochoa, X. M. E. 2008. Guía para producir cártamo INIFAP-CIRNO-CEVY-SEMAY en Sonora. Ciudad Obregón, Sonora. Folleto para productores núm. 38. 27 p.

Montoya, C. L. F.; Ochoa, B. J.; Wong, P. M.; Camarillo, P. y Macias, J. C. 2008. CIANO-OL, CIANO-LIN, RC-1002-L, RC-1005-L y RC-1033-L variedades de cártamo altamente tolerantes a falsa cenicilla (Ramularia carthami). INIFAP-CIRNO-CEVY. Cd. Obregón Sonora. Folleto técnico núm. 60. 27 p.

Montoya, C. L. y Ochoa, F. B. 2006. Guía para producir cártamo en el sur de Sonora. INIFAPCIRNO-CESS, Cd. Obregón Sonora. Folleto para productores núm. 37.24 p.

Montoya, C. L. 2010. El cultivo de cártamo (Carthamus tinctorius L.) en México. INIFAP-CIRNOCENEB. Cd. Obregón Sonora. Libro técnico núm. 5. 96 p.

Navejas, J. J. L.; Montoya, C. y Gutierres, E. P. 2008. Guía para producir cártamo en el Valle de Santo Domingo. BCS. INIFAP-CIRNO-SESD. Santo Domingo, Baja California Sur. Folleto para productores núm. 17. 17 p.

Navejas, J. J. J. A. C.; Navarro A. y Gutierres, E. P. 2014. Paquete tecnológico para la producción de cártamo. INIFAP-CIRNO-CETODS. La Paz, Baja California Sur. Folleto para productores núm. 19. 17 p.

Nikabadi, S. A.; Soleimani, S. M.; Dehdashti, S. M. and Yazdanibakhsh, M. 2008. Effect of sowing dates on yield and yield components of spring safflower (Carthamus tinctorius L.) in Isfahan Region. Pak. J. Biol. Sci. 11(5):1953-1956. Doi: 10.3923/pjbs.2008.1953.1956.

Padmavathi, P. and Virmani, S. M. 2013. Impact of climate change on safflower (Carthamus tinctorius L) in India and México. J. Agrometeorology. 15(1):58-61. 
Paquini, R. S. L. I.; Benítez, R. H. E.; Villaseñor, M. A.; Muñoz, O. y Vaquera, H. H. 2016. Incremento en el rendimiento y sus componentes bajo riego normal y restringido de variedades mexicanas de trigo. Rev. Fitotec. Mex. 39(4):367-378.

Pourad, S. 2008. Study on drought resistance indices in spring safflower. Acta Agronómica Hungarica. 56(2):203-212. https://doi.org/10.1556/AAgr.56.2008.2.9.

Quilantán, V. L. 1978. Cártamo. In: Cervantes, S. T. (Ed.). Recursos genéticos disponibles a México. Sociedad Mexicana de Citogenética, AC. 209-213 pp.

Ramírez, A. J. A. C. M.; Armenta, C. L.; Montoya, C. F.; Cabrera, C. y Valenzuela, J. R. B 2011. Epidemiologia de la falsa cenicilla del cártamo (Ramularia carthami) en el sur de Sonora. INIFAP-CIRNO-CENEB. Cd. Obregón Sonora. Folleto técnico núm. 82. 24 p.

Ramírez, A. J. A. F.; Cabrera, C. L.; Montoya, C. X. M.; Ochoa E. y Borbón, A. G. 2017. Efecto del riego en el desarrollo y control de la falsa cenicilla del cártamo en el sur de Sonora. INIFAP-CIRNO-CENEB. Cd. Obregón Sonora. Folleto técnico núm. 101. 28 p.

Ramonda, F. C. J.; Ferrero, Fritz, F. y Baudino, E. M. 2019. Influencia de la fecha de siembra sobre los determinantes fisiológicos y numéricos del rendimiento en el cultivo de cártamo (Carthamus tinctorius L.) en la pampa. SEMIARIDA. 29(1):63-69. https://doi.org/10.19137/semiarida.

Ramos, A. M. J. G.; Barrios, P. Z.; Hernández, A. J. y Hernández, O. 2019. Impacto de una posible depreciación del peso mexicano en las importaciones mexicanas de oleaginosas. Rev. Mex. Agronegocios. 44:208-220. Doi: 10.22004/ag.econ.292261.

REMAS. 2019. Red de Estaciones Meteorológicas Automatizadas del estado de Sonora. Reporte de estación. http://www.siafeson.com/remas/index.php/estacionglobal?rid=85.

Reta, S. D. G. J. S.; Serrato, C. H. M.; Quiroga, G. A.; Gaytán, M. y Figueroa, U. M. 2017. Secuencias de cultivo alternativas para incrementar el potencial forrajero y productividad del agua. Rev. Mex. Cienc. Pec. 8(4):397-406. http://dx.doi.org/10.22319/rmcp.v8i4.4645.

SAGARPA. 2017. Secretaría de Agricultura, Ganadería, Desarrollo Rural, Pesca y Alimentación. Oleaginosas mexicanas: canola, cártamo, girasol y soya. Planeación Agrícola Nacional 2017-2030. 26 p.

SIAP. 2021. Servicio de Información Agroalimentaria y Pesquera. Anuario estadístico de la producción agrícola. Servicio de Información Agroalimentaria y Pesquera-Secretaría de Agricultura y Desarrollo Rural. Ciudad de México. https://nube.siap.gob.mx/cierreagricola/.

Silveira, G. M. I. M. L.; Aldana, M. L. A.; Medina, J. y Serrano, A. E. 2009. Situación de la producción de cártamo (Carthamus tinctorius L.) en Sonora, México y factores asociados. Biotecnia. 11(3):44-56. http://dx.doi.org/10.18633/bt.v11i3.73.

Singh, N. A.; Garima, A. and Kapoor, R. 2019. Incidence and severity of fungal diseases of safflower in India. Crop Protection. 25. https://doi.org/10.1016/j.cropro.2019.104905.

Singh, S. S. V.; Angadi, K.; Grover, S.; Begna, S. and Auld, D. 2016. Drough response and yield formation of spring safflower under different water regimes in the semiarid Southern high Plains. Agricultural Water 163:354-362. https://doi.org/10.1016/j.agwat.2015.10.010.

Ullah, F. and Bano, A. 2011. Effect of plant growth regulators on yield and biodiesel production of safflower (Carthamus tinctorius L.). Brazilian J. Plant Physiol. 23(1):27-31. https://doi.org/10.1590/S1677-04202011000100005.

Valadez, G. J. y Cervantes, J. E. M. 2013. Guayalejo, variedad de cártamo para la región sur de Tamaulipas. INIFAP- CIRNE-CEHUAS. Villa Cuauhtémoc, Tamaulipas. Folleto técnico núm. MX-0-310302-52-03-14-09-29. 15 p. 
Valadez, G. J. y Cervantes, J. E. M. 2017. Promesa, variedad de polinización libre de cártamo para la región de las huastecas. Rev. Fitotec. Mex. 40(1):103-105.

Vosoughkia, M.; Hossainchi, G. L.; Ghavami, M. M.; Gharachorloo, M. and Delkhosh, B. 2012. Evaluation of oil content and fatty acid composition in seeds of different genotypes of safflower (Carthamus tinctorius L.). Inter. J. Agric. Sci. Res. 2(1):59-66.

Zapata, M. L. E. 2010. Situación y perspectivas del aceite de palma alto oleico O x G en Colombia. Palmas. 31(2):349-353. 